\title{
ВMJ Global Health Understanding the relationship between family planning method choices and modern contraceptive use: an analysis of geographically linked population and health facilities data in Haiti
}

To cite: Wang W, Mallick L. Understanding the relationship between family planning method choices and modern contraceptive use: an analysis of geographically linked population and health facilities data in Haiti. BMJ Glob Health 2019;4:e000765. doi:10.1136/ bmjgh-2018-000765

Handling editor Seye Abimbola

- Additional material is published online only. To view please visit the journal online (http://dx.doi.org/10.1136/ bmjgh-2018-000765).

Received 9 February 2018 Revised 30 August 2018 Accepted 31 August 2018

Check for updates

(C) Author(s) (or their employer(s)) 2019. Re-use permitted under CC BY-NC. No commercial re-use. See rights and permissions. Published by BMJ.

${ }^{1}$ The Demographic and Health Surveys (DHS) Program, Division of International Health and Development, ICF, Rockville, Maryland, USA

${ }^{2}$ The DHS Program, Avenir Health, Glastonbury,

Connecticut, USA

Correspondence to

Dr Wenjuan Wang;

wenjuan.wang@icf.com

\section{ABSTRACT}

Introduction This study linked data from the 2012 Haiti Demographic and Health Survey (DHS) and the 2013 Haiti Service Provision Assessment (SPA) to estimate the extent to which women's contraceptive use is associated with the method choices available in Haiti's health facilities.

Methods Using Global Positioning System (GPS) data for DHS clusters and for health facilities, we linked each DHS cluster to all of the family planning facilities located within a specified distance, and then measured the cluster's level of contraceptive method choice based on the number of facilities within the buffer zone that offered three or more modern contraceptive methods. Random intercept logistic regressions were used to model the variation in individual modern contraceptive use and the availability of multiple method choices at the cluster level.

Results Limited number of family planning facilities in Haiti offered at least three modern contraceptive methods (51\% in urban and $23 \%$ in rural). Seventeen percent of both rural and urban women lived in an area with low availability of multiple methods-meaning that no facility in the specified buffer zone offered three or more contraceptive methods. Another $29 \%$ of rural women and $41 \%$ of urban women had medium availability - that is, only one facility in the buffer zone offered three or more methods. In rural areas, compared with women living in a cluster with low availability of multiple methods, the odds of using a modern method are $73 \%$ higher for women living in a cluster with medium availability, and over twice as high for women living in a cluster with high availability. A similar positive relationship was also found in urban areas.

Conclusions Women in Haiti have only limited proximity to a health facility offering a variety of contraceptive methods. Improving access to a range of methods available at health facilities near where people live is critical for increasing contraceptive use in both urban and rural areas of Haiti.

\section{INTRODUCTION}

Family planning is critical for reducing maternal mortality and improving maternal

\section{Key questions}

What is already known?

- Extant research on the association between family planning service environment and contraceptive use has mostly concentrated on sub-Saharan Africa; little has been done in Haiti, a country with the lowest use of modern contraception in the region.

\section{What are the new findings?}

- Haitian women of reproductive age in both rural and urban areas outside the Port-au-Prince metropolitan area have limited proximity to a health facility offering multiple contraceptive method choices.

- Availability of a nearby health facility that offers three or more contraceptive methods is significantly associated with women using a modern contraceptive method in both rural and urban areas.

What do the new findings imply?

- Improving access to a range of methods available at health facilities near where people live is critical for increasing contraceptive use in both urban and rural areas of Haiti.

and child health outcomes. ${ }^{1-4}$ Despite the efforts of family planning programmes worldwide, unmet need for family planning is only slowly declining in many resource-constrained settings. ${ }^{5}$ One of the most commonly cited reasons for non-use of contraception is lack of access-which has been characterised as a 'supply-side' issue, which health facilities and providers have the potential to address and resolve. ${ }^{6}$ In fact, central to Bruce-Jain framework of quality of care developed in 1990 is the first domain 'Choice of Methods'.? However, empirical research on this relationship is limited. ${ }^{8}$

Research has not yet identified the optimal mix of contraceptive methods to maximise contraceptive use. ${ }^{9}$ Theoretically, however, 
the availability of a range of contraceptive methods at health facilities provides a woman with more options from which to choose a method that meets her needs-therefore leading to adoption and continued use of contraception. A woman's contraceptive needs and choices can vary based on whether she wants to space births or limit them altogether, whether she is currently breastfeeding, spousal preferences or cultural acceptability of contraceptive use and whether she may need to use a method discreetly, whether she prefers hormonal or non-hormonal methods, and her experience of side-effects. Further, her selection of a method is constrained by the methods available to her. For example, long-acting methods require higher levels of provider and facility capacity, which may be limited in resource-poor settings. ${ }^{5}$

Ross and Stover used the Family Planning Effort Index, ${ }^{10}$ which scores family planning programme in low-income settings based on their policies, services, environment and access to a number of methods, ${ }^{11}$ to examine whether method access is associated with modern contraceptive prevalence in 113 countries. They found that an increase in the number of methods available was associated with an increase in modern contraceptive prevalence. An earlier study that also used programme effort scores as a proxy for method availability in 64 developing countries found that contraceptive prevalence rose with increased availability to four modern methods (female sterilisation, the intrauterine device (IUD), the pill and the condom).$^{12}$ Additionally, women who are using longacting or reversible contraceptives have a significantly lower risk of discontinuing while still in need of family planning if they live in areas with higher-quality of family planning services. ${ }^{13}$

As a growing number of health facility surveys are conducted on a routine basis, and as geographical data become available both in surveys of health facilities and household surveys, it is possible to measure the association between method availability and contraceptive use by geographically linking health facility survey data to household-based survey data. Only a handful of analyses have used this approach, however, and their findings vary. ${ }^{14-17}$ In one study linking household data from the Demographic and Health Surveys (DHS) and health facility data collected through the Service Provision Assessment (SPA), Wang and colleagues analysed family planning data from four East African countries, finding that at the regional level an increase in the average number of contraceptive methods available was associated with higher odds of modern contraceptive use. ${ }^{17}$ In another study linking DHS and logistics management information system (LMIS) data, Skiles and colleagues showed that both distance from a family planning facility as well as availability of a reliable supply of methods were associated with contraceptive use in Malawi. ${ }^{15}$ Tumlinson et al found only a marginally significant relationship between an available supply of a mix of family planning methods and the level of contraceptive use in Kenya. ${ }^{16}$ Conversely, Gage and Zomahoun used geographically linked data collected in Nigeria during the 2009 Community Participation for Action in the Social Sectors project and found that having an above-average number of family planning methods was not significantly associated with modern contraceptive use within a local government area. ${ }^{14}$

While research on the family planning service environment and contraceptive use has centred on sub-Saharan Africa, none has been done in Haiti, a country with the highest level of unmet need for family planning and lowest use of modern contraception in the Latin America and the Caribbean region. ${ }^{18}$ Only about a fifth $(22 \%)$ of Haitian women of reproductive age use a modern method, while over a third (35\%) of married women who want to limit or space births are not using any contraceptive method. ${ }^{19}$ Among modern contraception users, method mix is highly skewed. Injectables dominate the method mix, accounting for nearly two-thirds $(62 \%)$ of modern contraceptive users among married women. ${ }^{19} 20$

Service delivery has been a serious concern in Haiti, given its impoverished state. ${ }^{21}$ The 2010 earthquake decimated the already overburdened health system. Much of the country's health infrastructure was destroyed and many health service providers died..$^{1822-24}$ The earthquake exacerbated the pre-existing barriers to care that women have faced in Haiti: poor infrastructure, high healthcare costs and geographic distance to facilities, particularly in rural areas. ${ }^{24}$ Haitians have very limited access to high-quality care, especially in rural areas. ${ }^{14}$ Further, the mountainous topography and the lack of paved roads have been a barrier to obtaining care. ${ }^{25} 26$ In large part, both before the earthquake but increasingly so after the earthquake, health services including family planning are provided by the private sector. ${ }^{18}$ However, bilateral and multilateral organisations have dedicated significant resources towards regenerating the public system, establishing mechanisms for maintaining a contraceptive commodities supply as well as supporting a community health agents programme. ${ }^{18}$ In fact, donor funding contributes to almost two-thirds of total health expenditures. ${ }^{27}$ Despite these efforts, stock-out of commodities is still common. A United Nations Population Fund (UNFPA) assessment of service delivery points demonstrated a decrease in facilities reporting 'no stock-outs' from 53 in 2010 to $26 \%$ in $2013 .^{28}$

Still, little is known about the impact of the supply of modern contraceptive methods on use of family planning in Haiti. While it is possible that women obtain short-acting contraceptive methods, such as condoms and oral contraceptives, from pharmacies, independent sellers or other informal markets, we hypothesise that women would be more likely to use family planning methods if nearby health facilities had more methods available. Thus, this study aims to test this hypothesis and fill the gap in the literature on the relationship between availability of modern family planning methods at health facilities and contraceptive use in Haiti. 


\section{METHODS}

\section{Data}

The study used data from the 2012 Haiti DHS and the 2013 Haiti SPA. The DHS is a nationally representative household survey that provides estimates for a range of health indicators, including contraceptive use among women age 15-49, as well as their sociodemographic characteristics. The SPA survey provides information on family planning services at health facilities. Geographic coordinates data collected in both Haiti surveys were used to link DHS clusters and SPA facilities. Using a two-stage cluster sampling approach, the DHS survey selected 445 clusters at the first stage and a sample of 13181 households at the second stage. Additional information on the sample design can be found in the final survey report. ${ }^{19}$ The survey approached all women age 15-49 in the selected households for individual interview in which individual sociodemographic characteristics and information on use of health services were collected. With a response rate of $98 \%$, a total number of 14287 women participated in the interview.

The 2012 Haiti DHS collected geographic coordinates of the sampled clusters. To protect confidentiality of the participants, coordinates data were geographically displaced before release to the public. The displacement distance was up to $2 \mathrm{~km}$ for urban clusters and $5 \mathrm{~km}$ for rural clusters, with a further randomly selected $1 \%$ of rural clusters displaced up to $10 \mathrm{~km} .^{29}$ Our analysis excluded eight clusters with missing coordinates data and 45 camp clusters that temporarily housed the population displaced by the 2010 earthquake. Women interviewed in camp clusters were likely to reside in a different location at the time when they needed family planning services; therefore, the family planning service environment was not the same as where they were surveyed. Women may have obtained camp-based services but those are likely to be informal and thus not assessed by the SPA survey.

We stratified the analysis by urban-rural residence because of substantial differences between urban and rural areas in the health service environment and in people's healthcare seeking behaviours. ${ }^{14} 19$ For urban areas, we excluded the Port-au-Prince metropolitan area (Portau-Prince and urban zones of the Ouest region), since research has shown that the linkage approach used in this study is not appropriate for such areas with high density of DHS clusters and health facilities. ${ }^{26}$ Therefore, urban areas referred to in this paper represent non-metropolitan urban areas. ${ }^{\text {i }}$ We excluded unmarried women from the analysis as only $15 \%$ of unmarried women are sexually active and their primary contraceptive method is condom obtained from informal sources as opposed to health facilities. With the study restricted to married women, this yielded an analysis sample of 3826 women from 241 rural clusters and 1216 women from 85 urban clusters. Figure 1 shows the location of the clusters included in the analysis and the location of family planning facilities covered in the SPA.

The 2013 Haiti SPA is a census of all the formal-sector health facilities in Haiti. Of the 905 health facilities interviewed, 756 reported providing family planning services. The Haiti SPA collected data through four types of questionnaires: facility inventory, health provider interview, observation of consultations and client exit interview. Facility data used for this analysis come primarily from the facility inventory, which was administered to the facility manager or the most knowledgeable person for specific service areas.

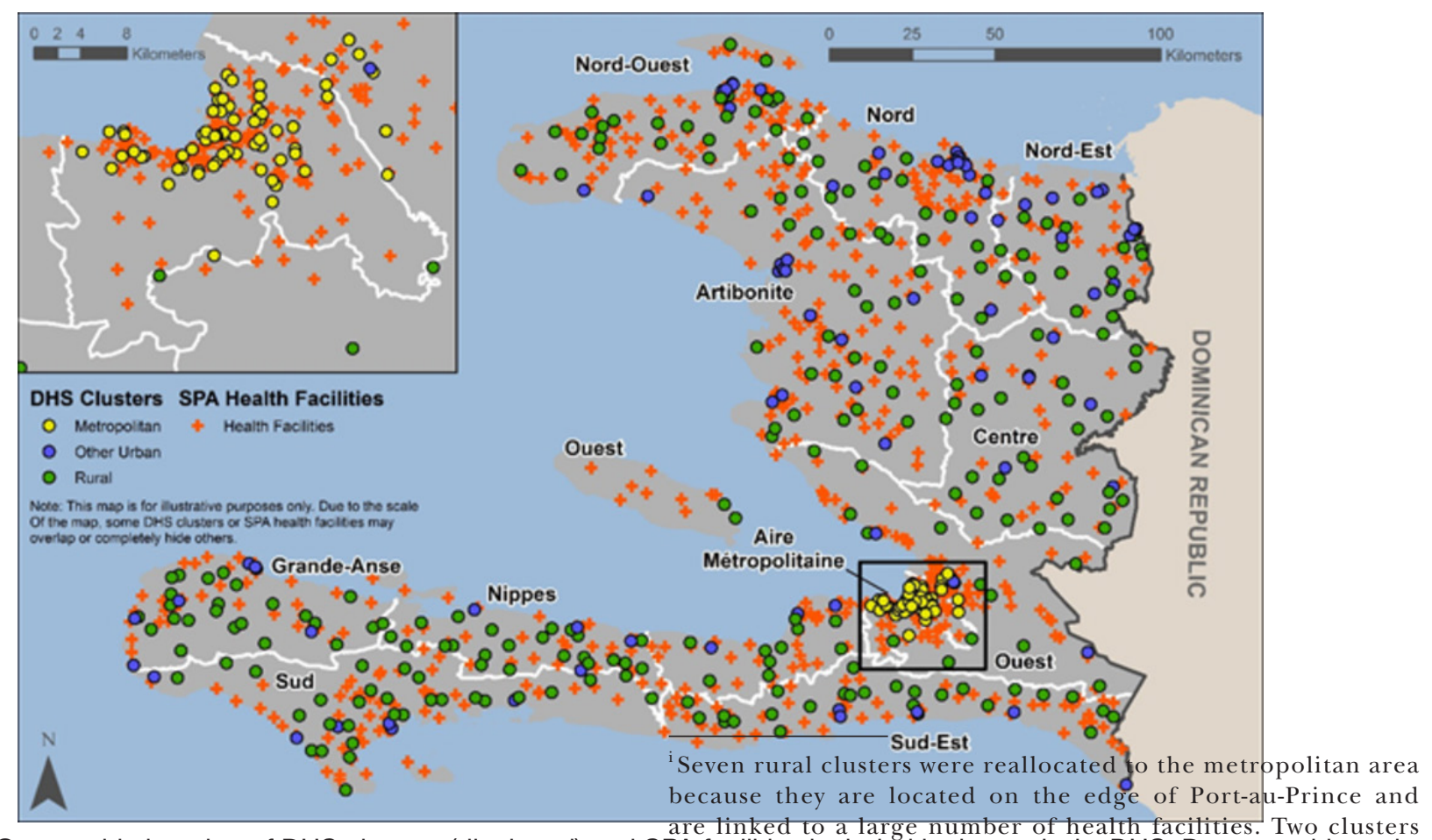

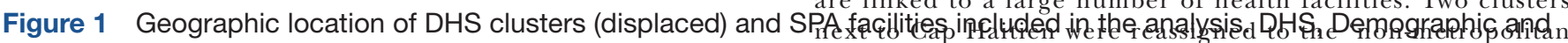
Health Survey; SPA, Service Provision Assessment.

urban' group for a similar reason. 
For family planning services, the SPA facility inventory collected data on contraceptive method mix and method availability, presence of infrastructure, equipment, guidelines and infection control items necessary for providing good-quality family planning services. The Haiti SPA also collected coordinate location of each interviewed health facility. These data were released without displacement.

\section{Linking DHS clusters and SPA facilities}

Access to family planning is largely determined by the services available in nearby facilities. Because of the displacement of clusters, it is not possible to link a cluster to the nearest facility because the closest facility identified based on the released geographic data may not actually be the nearest facility; therefore, such linkage may be subject to substantial misclassification and errors. ${ }^{15}$ In addition, such linkage is problematic when individuals bypass the nearest facility due to such concerns as the quality of care or cost. In this study, instead of looking at the nearest facility, we analysed all health facilities within a specific distance buffer from the cluster and used data on the linked facilities as a proxy of the service environment of the cluster. ${ }^{26}$ This approach assumed that women would use health facilities within the specified buffer. The distance buffer, $5 \mathrm{~km}$ from urban clusters and $10 \mathrm{~km}$ from rural clusters, was chosen based on the DHS recommendation in using GPS data. ${ }^{30}$ Using the straight-line distance between DHS clusters and SPA facilities, we identified all facilities within the specified buffer of each cluster. Information on linked facilities was summarised to measure the provision of family planning services around the cluster.

\section{Data analysis}

We used multilevel (individual-level and cluster-level) random intercept logistic regressions to model the association between a woman's use of modern contraception and the availability of family planning methods at nearby health facilities. Such models were used to adjust for the clustering effect, given that women from the same cluster may share certain characteristics. Moreover, random intercept models allow us to differentiate the variation in outcome explained by individual-level and cluster-level factors. ${ }^{31}$ The outcome variable was use of any modern contraceptive method, including female sterilisation, male sterilisation, pill, IUD, injectables, implants, lactational amenorrhea method, emergency contraception and standard days method. Condom users (both female and male condoms) were excluded from the analysis because many respondents report obtaining condoms at sources outside of the facilities that a SPA survey usually captures. Among women who reported male condoms as their method of contraception, $44 \%$ reported their most recent source of condoms was shops, mobile clinics, fieldworkers or friends/relatives. ${ }^{19}$

The key predictor was availability of a choice of multiple methods at nearby health facilities that provide family planning services. This variable was measured at the cluster level and computed using health facility data. In the Haiti SPA, all facilities offering family planning services were asked if the facility provides, prescribes or counsels on female sterilisation, male sterilisation, pill, IUD, injectables, implants, condom, emergency contraception or lactational amenorrhea method and if the facility has valid stock on the day of the assessment for some methods such as pills, injectables, implants or emergency contraception observed on-site. In this way, for each facility, the total number of modern methods provided was determined. Again, condoms were excluded. After identifying facilities within a buffer of $10 \mathrm{~km}$ for rural clusters and $5 \mathrm{~km}$ for urban clusters, we counted the number of facilities offering three or more methods and used this number to classify clusters into three groups: low, medium and high level of availability of multiple method choices. We chose to use three or more methods as the cut-off because few facilities had four or more methods and having at least three methods represent a reasonable range of method choices at health facilities. In fact, we found that almost all facilities with three or more methods had at least one long-acting reversible contraception. Clusters linked to no facilities providing three or more methods within the buffer were classified in the low-availability group; those linked to one such facility were classified in the medium-availability group and the rest, which were linked to more than one facility offering three or more methods, were classified in the high-availability group. The three-level classification was determined based on the distribution of clusters according to the number of facilities within the buffer that offer three or more contraceptive methods. This classification differentiates clusters that have no facility, one or some facilities offering a mix of modern contraceptive methods.

The regressions adjusted for covariates that the literature has shown to be associated with contraceptive use. These include women's age, number of living children, education, occupation, household wealth status, religion, exposure to family planning messages in the media and recent contact with family planning providers. ${ }^{32-35}$ Exposure to family planning messages was defined as hearing about family planning in past few months on radio, TV or in newspaper/magazine. A woman was considered having recent contact with family planning providers if she was visited by a family planning provider or discussed family planning when she visited a health facility in past 12 months.

\section{RESULTS}

Of the health facilities found to provide family planning services in Haiti, the majority were in rural areas. Table 1 shows the distribution of these facilities by type of facility, managing authority and department in urban and rural areas. In rural areas, nearly two-thirds (62\%) of facilities that provided family planning services were dispensaries, and about a quarter (23\%) were health centres without beds, while hospitals were only $3 \%$ of the total. In urban areas, the composition of family planning facilities was more evenly distributed among different facility types. In both rural and urban areas, most facilities were managed 
Table 1 Characteristics of health facilities that provide family planning services, Haiti SPA 2013

\begin{tabular}{|c|c|c|c|c|}
\hline \multirow[b]{2}{*}{ Characteristics } & \multicolumn{2}{|l|}{ Urban } & \multicolumn{2}{|l|}{ Rural } \\
\hline & $\%$ & $\mathbf{N}$ & $\%$ & $\mathbf{N}$ \\
\hline \multicolumn{5}{|l|}{ Type of facility } \\
\hline Hospital & 29.3 & 44 & 3.3 & 16 \\
\hline Health centre with beds & 22.6 & 34 & 12.0 & 58 \\
\hline Health centre without beds & 33.5 & 50 & 22.6 & 109 \\
\hline Dispensary & 14.6 & 22 & 62.1 & 300 \\
\hline \multicolumn{5}{|l|}{ Managing authority } \\
\hline Government & 53.4 & 80 & 45.6 & 220 \\
\hline NGO/private not-for-profit & 9.4 & 14 & 14.7 & 71 \\
\hline Private for-profit & 13.3 & 20 & 17.0 & 82 \\
\hline Mixed $^{*}$ & 23.9 & 36 & 22.8 & 110 \\
\hline \multicolumn{5}{|l|}{ Department } \\
\hline $\begin{array}{l}\text { Aire metropolitaine/ Reste- } \\
\text { Ouest }\end{array}$ & 13.3 & 20 & 21.9 & 106 \\
\hline Sud-Est & 6.6 & 10 & 9.5 & 46 \\
\hline Nord & 15.3 & 23 & 8.9 & 43 \\
\hline Nord-Est & 3.3 & 5 & 5.8 & 28 \\
\hline Artibonite & 8.6 & 13 & 19.0 & 92 \\
\hline Centre & 6.6 & 10 & 5.4 & 26 \\
\hline Sud & 20.6 & 31 & 6.6 & 32 \\
\hline Grand-Anse & 10.0 & 15 & 5.0 & 24 \\
\hline Nord-Ouest & 8.9 & 13 & 14.2 & 68 \\
\hline Nippes & 6.6 & 10 & 3.9 & 19 \\
\hline Total & $100 \%$ & 150 & $100 \%$ & 483 \\
\hline
\end{tabular}

${ }^{*}$ Mixed facilities are non-profit facilities that also receive subsidies or salaried staff from the government.

SPA, Service Provision Assessment.

by the government or by a mixed government and private non-profit managing authority.

Family planning facilities in Haiti reported providing a range of modern contraceptive methods; the most commonly reported methods were pills, injectables, male condoms and implants (table 2). Overall, $77 \%$ or more of the facilities provided pills, injectables and male condoms. In urban areas, $42 \%$ of family planning facilities reported having implants available, over twice the percentage of facilities in rural areas $(20 \%)$ that reported this method. Overall, $51 \%$ of family planning facilities in urban areas and $23 \%$ in rural areas provided at least three methods (with condom excluded). Figure 2 shows the percentage of facilities with at least three modern methods available, by facility type and managing authority. The highest level of availability was in hospitals: $67 \%$ of hospitals provided at least three methods, followed by health centres with beds, at $45 \%$. Dispensaries had the lowest availability, at $20 \%$. By managing authority, the percentage providing three or more methods was highest in government facilities (33\%) and lowest in private for-profit facilities $(18 \%)$.
Table 2 Percentage of family planning facilities in Haiti that provide specific modern contraceptive methods*, Haiti SPA 2013

\begin{tabular}{lcr}
\hline $\begin{array}{l}\text { Modern contraceptive } \\
\text { method }\end{array}$ & Urban & Rural \\
\hline Pill & 82.0 & 77.6 \\
\hline Injectables & 81.3 & 77.8 \\
\hline Male condom & 88.7 & 80.3 \\
\hline Implants & 41.9 & 15.3 \\
\hline Female condom & 6.0 & 6.4 \\
\hline IUD & 5.3 & 0.6 \\
\hline Emergency contraception & 8.6 & 3.9 \\
\hline Standard days method & 19.9 & 12.0 \\
\hline Male sterilisation & 12.0 & 2.5 \\
\hline Female sterilisation & 17.3 & 2.7 \\
Provide three or more methods & 50.5 & 23.1 \\
(excluding condom) & & \\
Total number of facilities & 150 & 483 \\
\hline
\end{tabular}

*Facility provides, prescribes or counsels clients on family planning methods and facility has valid products observed onsite if method is pill, injectables, implants, male condom, female condom and emergency contraception.

IUD, intrauterine device; SPA, Service Provision Assessment.

In the 2012 Haiti DHS, after excluding camp clusters and clusters with missing GPS data, 3826 women from 241 rural clusters and 1216 women from 85 urban clusters remained in the analysis. Among the linked facilities within the buffer, we counted the number that provided at least three family planning methods and used this number to categorise clusters. Figure 3 shows the distribution of women by the availability of multiple method choices at nearby facilities. In rural areas, $17 \%$ of women lived in an area with low availability of multiple methods, meaning that no facility with $10 \mathrm{~km}$ from the cluster offered at least three modern methods, $29 \%$ of women had medium availability and $54 \%$ had high availability. In urban areas, $42 \%$ of women lived in an area with a high availability of choices-that is, where two or more nearby facilities reported availability of at least three contraceptive methods.

Overall, 5042 married women were analysed for their use of modern contraception. Background characteristics of these women in each geographic location are shown in online supplementary table 1 . Modern contraceptive prevalence (except condom use) was $30 \%$ in both urban and rural areas. Figure 4 highlights the relationship between women's use of modern contraception and the availability of multiple family planning methods at nearby facilities. In rural areas, modern contraceptive use was higher in areas that had more facilities with multiple modern methods available. In areas where facilities had a high level of availability-offered at least three methods- $35 \%$ of women reported current use of modern contraception compared with only $20 \%$ of women in clusters where no facility within the service environment offered three or more methods. In 

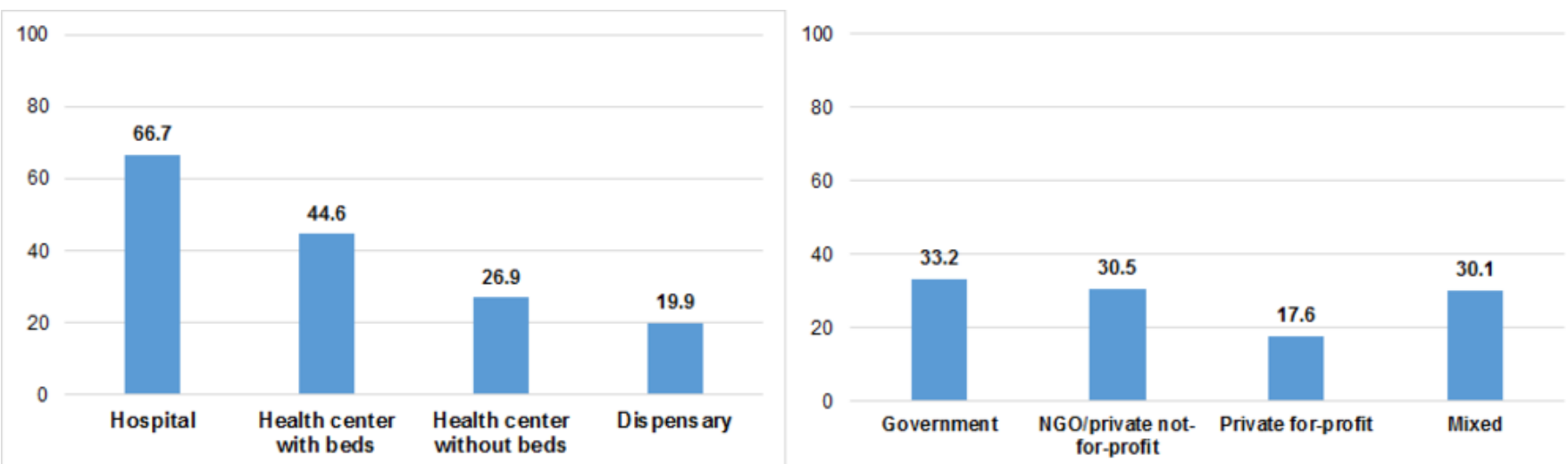

Figure 2 Percentage of family planning facilities providing at least three modern contraceptive methods, by facility type and managing authority, Haiti SPA 2013. SPA, Service Provision Assessment.

urban areas, women living in clusters with medium or high availability reported higher use of contraception compared with women in low-availability clusters.

Using multilevel multivariable logistic regression models, we assessed how women's contraceptive use is associated with the availability of multiple method choices within the service environment. We ran separate models for urban and rural. Table 3 presents odds ratio (OR) and $95 \%$ confidence interval (CI) for the level of availability of multiple methods as well as for the covariates adjusted for in the models. Random-effect is significant in both models, indicating significant variations in outcome at the cluster level. In rural areas, the level of method choice is positively and significantly associated with women's use of modern contraception after controlling for women's characteristics. Compared with women in clusters with a low level of availability of multiple methods, the odds of using a modern contraceptive method are $73 \%$ higher for women in a cluster with a medium level of availability (OR, 1.73; 95\% CI 1.23 to 2.45), and over twice as high for women in a cluster with a high level of availability (OR, 2.23; 95\% CI 1.61 to 3.09). A similar positive relationship is also found in urban areas. Among women's background characteristics, age, number of living children and contact with a family planning provider are consistently associated with the use of contraception in both urban and rural areas. In contrast,

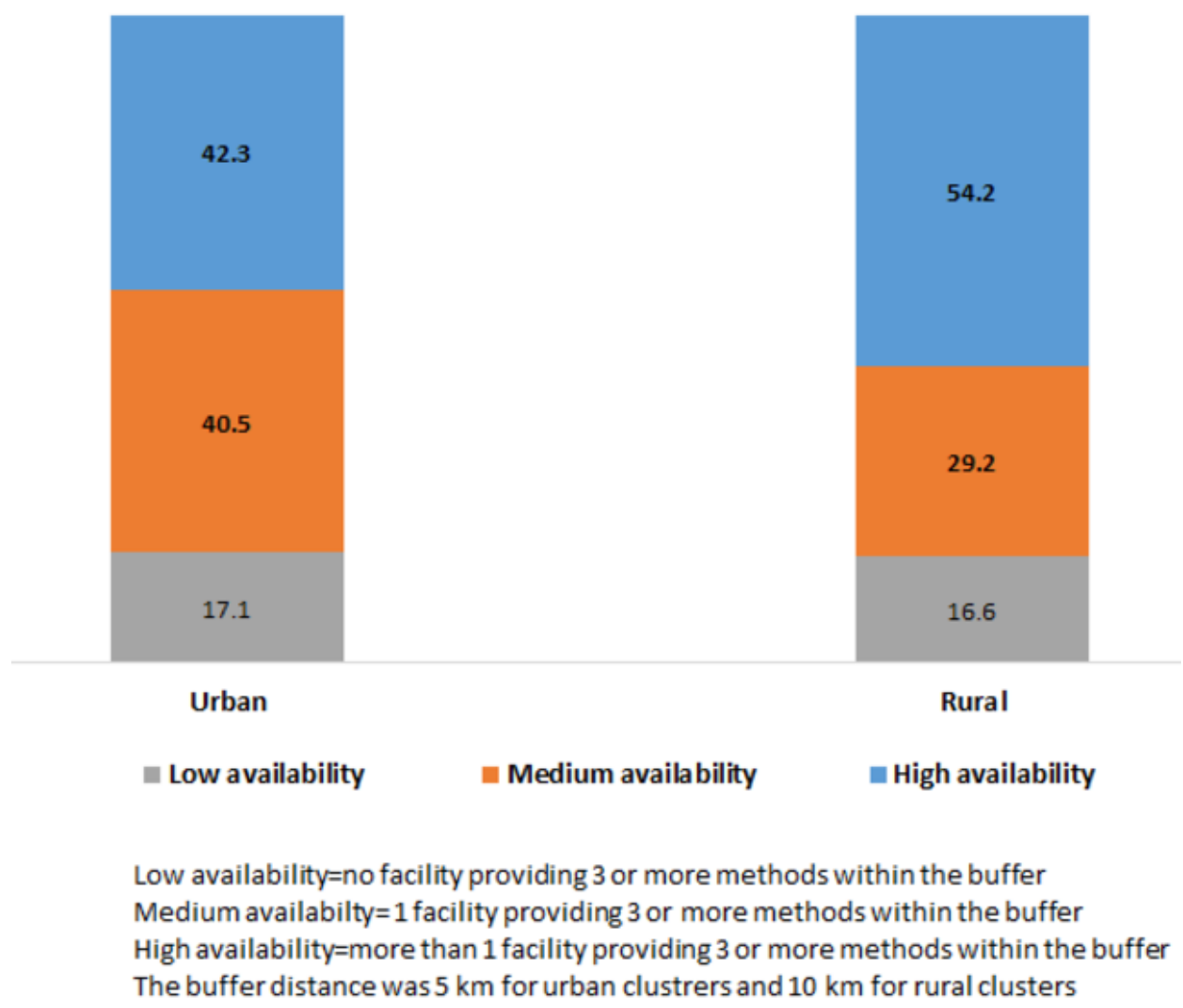

Figure 3 Percent distribution of married women in Haiti by availability of multiple method choices at nearby family planning facilities. 


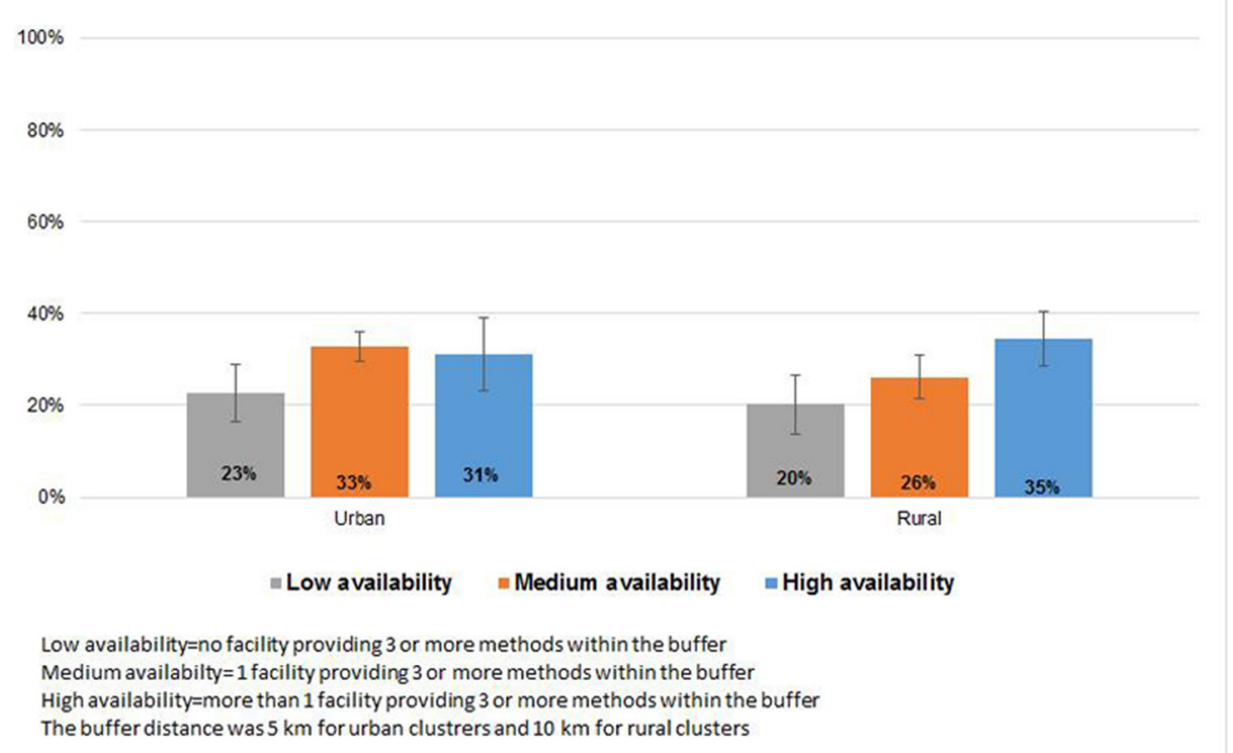

Figure 4 Women's use of modern contraceptive methods in Haiti by availability of three or more methods at nearby facilities and residence.

women's household wealth status and religion do not seem to be important predictors of modern contraceptive use.

\section{DISCUSSION}

Linking data from the 2012 Haiti DHS, a nationally representative household survey, and the 2013 Haiti SPA, a health facility survey, this study examined the relationship between women's contraceptive use and the family planning service environment, specifically the availability of multiple method choices at nearby health facilities. The results indicate a strong association between the availability of three or more methods and women's use of modern contraception in both urban and rural areas. The presence of at least one facility offering a range of methods within a reasonable distance from the community (cluster) was significantly associated with increased use of modern contraceptive methods. Such association is evident in both rural and non-metropolitan urban areas.

The availability of contraceptive options impacts women's contraceptive use choices and determines the method mix among users. This is observed in the recent shift from male condom and pills to injectables and implants among contraceptive users in many sub-Saharan African countries due to the considerable donor support in increasing the supply and availability of these two methods in this region. ${ }^{36}$ In Central Africa countries where there is still limited availability of other methods, male condoms remain as the dominant contraceptive method among both married and unmarried women. ${ }^{37}$ A study in Uganda showed that proximity to a private health facility was associated with a greater likelihood of contraceptive use in urban areas but not in rural areas. ${ }^{38}$ Although not explored by the authors of this study, it is possible that health facilities in rural areas had limited contraceptive options and therefore being close to a health facility did not help address women's contraceptive needs.

Haiti heavily relies on external support for most funding of health services and the investment in family planning has been increasing but still limited. ${ }^{27} \mathrm{We}$ found although the majority of health facilities provide family planning services, choice of modern methods of contraception at health facilities is limited-only half of the family planning facilities in urban areas and less than a quarter of facilities in rural areas provide three or more methods. The communities studied had limited choices in nearby facilities, especially in rural areas where $17 \%$ of women lived in a community where no facility within $10 \mathrm{~km}$ offered three or more contraceptive methods. The availability of implants, IUD and sterilisation is very limited in rural family planning facilities. This could be due to a number of factors, including shortages of supplies, commodities, trained providers to perform the procedure or other deficits in the health system.

Providing a range of family planning methods from which to choose is important to meet the needs of couples at different life stages and also to allow users to switch methods, thereby increasing long-term use of contraception. ${ }^{7}$ Our findings suggest that family planning programmes in Haiti need to focus on increasing method diversity in both urban and rural areas. More than $80 \%$ of health facilities in rural areas that provide family planning services are dispensaries or health centres without beds. These two types of facilities reported the lowest availability of multiple methods. To increase contraceptive use in rural areas, health centres without beds and dispensaries need to be better supplied with a range of contraceptives. For remote 
Table 3 Results of multivariable logistic regressions of use of modern contraceptive methods among married women age $15-49$ in Haiti

\begin{tabular}{|c|c|c|c|c|}
\hline \multirow[b]{2}{*}{ Variables } & \multicolumn{2}{|l|}{ Urban } & \multicolumn{2}{|l|}{ Rural } \\
\hline & OR & $95 \% \mathrm{Cl}$ & OR & $95 \% \mathrm{Cl}$ \\
\hline \multicolumn{5}{|c|}{ Level of availability of multiple family planning methods } \\
\hline Low availability & 1.00 & & 1.00 & \\
\hline Medium availability & $1.77^{\star \star}$ & 1.19 to 2.63 & $1.73^{\star *}$ & 1.23 to 2.45 \\
\hline High availability & $2.01^{* *}$ & 1.33 to 3.05 & $2.23^{\star}$ & 1.61 to 3.09 \\
\hline \multicolumn{5}{|l|}{ Age } \\
\hline $15-24$ & 1.00 & & 1.00 & \\
\hline $25-34$ & 0.78 & 0.54 to 1.13 & 1.04 & 0.83 to 1.31 \\
\hline $35-44$ & $0.52^{\star \star}$ & 0.33 to 0.81 & 0.81 & 0.61 to 1.06 \\
\hline $45-49$ & $0.25^{\star}$ & 0.13 to 0.47 & $0.29^{*}$ & 0.20 to 0.42 \\
\hline \multicolumn{5}{|l|}{ Number of living kids } \\
\hline $0-1$ & 1.00 & & 1.00 & \\
\hline $2-3$ & $2.10^{\star}$ & 1.54 to 2.86 & $2.70^{\star}$ & 2.15 to 3.38 \\
\hline $4-5$ & $1.78^{\star *}$ & 1.15 to 2.75 & $3.32^{*}$ & 2.52 to 4.37 \\
\hline $6+$ & $2.27^{\star \star}$ & 1.23 to 4.20 & $3.19^{*}$ & 2.32 to 4.38 \\
\hline \multicolumn{5}{|l|}{ Education } \\
\hline None & 1.00 & & 1.00 & \\
\hline Primary & 1.08 & 0.71 to 1.64 & 1.09 & 0.91 to 1.31 \\
\hline Secondary or higher & 1.02 & 0.65 to 1.61 & 1.13 & 0.87 to 1.46 \\
\hline \multicolumn{5}{|l|}{ Occupation } \\
\hline Unemployed & 1.00 & & 1.00 & \\
\hline Agriculture & 1.07 & 0.57 to 2.02 & 1.27 & 0.98 to 1.63 \\
\hline Professional & 1.23 & 0.93 to 1.63 & $1.28^{\star *}$ & 1.08 to 1.52 \\
\hline Other & 1.76 & 0.97 to 3.18 & 1.17 & 0.67 to 2.04 \\
\hline \multicolumn{5}{|l|}{ Wealth Quintile† } \\
\hline Lowest & na & & 1.00 & \\
\hline Second & na & & 0.93 & 0.78 to 1.11 \\
\hline Middle & na & & 1.01 & 0.80 to 1.29 \\
\hline Lowest-Middle & 1.00 & & na & \\
\hline Fourth & 0.86 & 0.63 to 1.18 & 1.22 & 0.83 to 1.79 \\
\hline Highest & $0.64^{\star \star \star}$ & 0.43 to 0.93 & 0.77 & 0.40 to 1.46 \\
\hline \multicolumn{5}{|l|}{ Religion } \\
\hline Catholics & 1.00 & & 1.00 & \\
\hline Protestant & 0.79 & 0.61 to 1.02 & $0.78^{\star \star}$ & 0.67 to 0.92 \\
\hline Vaudousant/other & 0.58 & 0.32 to 1.05 & 0.94 & 0.70 to 1.25 \\
\hline \multicolumn{5}{|c|}{ Exposure to family planning messages } \\
\hline No exposure & 1.00 & & 1.00 & \\
\hline Weekly or more & 0.91 & 0.71 to 1.17 & 1.03 & 0.88 to 1.20 \\
\hline \multicolumn{5}{|c|}{ Contact with family planning provider } \\
\hline No & 1.00 & & 1.00 & \\
\hline Yes & $1.89^{\star}$ & 1.45 to 2.46 & $1.82^{*}$ & 1.55 to 2.13 \\
\hline Random parameter & 0.06 & & 0.10 & \\
\hline Number of women & 1216 & & 3826 & \\
\hline Number of clusters & 85 & & 241 & \\
\hline
\end{tabular}

${ }^{* * *} \mathrm{P}<0.001 ;{ }^{* *} \mathrm{P}<0.01 ;{ }^{*} \mathrm{P}<0.05$.

†In non-metropolitan urban areas, very few women were in the lowest and second wealth quintiles, and they were combined into the middle wealth group. 
areas where health facilities are sparse, mobile clinics and community-based services could help improve access to family planning. While having a range of methods available is essential for method choices, other aspects from the supply perspective that are not covered in this study are also important. Cost of services can prevent women from obtaining family planning services they need. Research found that contraceptive use in low-income countries increases with the share of government facilities that tend to provide services with low cost or no cost. ${ }^{39}$ While long-acting reversible methods are cost-effective in the long term, it can be challenging for individuals to pay the upfront cost of the services. ${ }^{40}$ Although in Haiti public health facilities are mandated to provide free family planning service, cost could still be a barrier to contraceptive use when women have to use the private sector due to the lack or stock-out of methods in public facilities. ${ }^{41}$ Provision of quality family planning services is also key to the contraceptive uptake and continuation. ${ }^{42}{ }^{43}$ Previous research found that quality of family planning counselling in health facilities in Haiti remains a concern. ${ }^{44}$

The methodological strength of this paper is linking health facilities and communities with GPS data using a buffer linkage approach. The availability of GPS data for DHS clusters and health facilities provides opportunities for linking information for households and health facilities. Among the limited studies based on DHS surveys and SPA or other health facility surveys to examine the relationship between service provision and use, most linked DHS clusters to the nearest health facility and analysed how physical access to health facilities-distance to the nearest facility and/or the services provided at the health facility-affects the use of health services. This approach using distance as a measurement of access to healthcare is prone to errors resulting from the geographical displacement of DHS clusters.$^{15}$ The use of the nearest facility to define the service environment is problematic for several reasons. In Haiti, we found that within the specific buffer, $40 \%-50 \%$ of clusters in rural and urban areas were linked to more than one facility offering three or more family planning methods. In such circumstances, the displacement could cause one of these facilities to be identified as the closest facility even though it is not, and therefore its service provision does not represent the service environment nearest to the community. Furthermore, even when the nearest facility is correctly identified, linking clusters to the nearest facility does not account for the fact that women may bypass the nearest facility in favour of seeking better-quality services further away. Thus, we consider it more prudent to examine the average service provision of all health facilities within an accessible distance from the community. The buffer distance chosen $(10 \mathrm{~km}$ in rural areas and $5 \mathrm{~km}$ in urban areas) reduced misclassification errors due to cluster displacement. All facilities in urban and most in rural within the maximum displacement radius around the real location of the cluster were captured (given that urban clusters were displaced up to $2 \mathrm{~km}$ and most rural clusters were displaced up to $5 \mathrm{~km}$ ).

One limitation of this study is the gap in time between the outcome variables and the service variables. Facility data reflect the current service environment at the time of the 2013 Haiti SPA. Although the question on women's contraceptive use in the 2012 DHS was framed as 'current', the service environment when women needed the service could be different from that during the interview. For instance, stockouts of contraceptive methods could have occurred. Associating SPA data on service provision and DHS data on contraceptive use could be problematic if the service environment changed substantially over the time between the two surveys. Second, the use of informal health outlets has a potentially confounding effect. SPA data reflect services provided at formal healthcare facilities, but many people use informal sectors for healthcare. In Haiti, the majority of male condom users interviewed in the DHS obtained condoms from shops or friends or relatives rather than healthcare facilities; therefore, we had to exclude condom use from the analysis. A large proportion of pill users in Haiti also rely on non-facility resources for their supplies such as pharmacies, mobile clinics and shops, none of which are captured in the SPA survey. Finally, we identified facilities around DHS clusters based on a straight-line distance measurement. This approach did not take into account the mountainous terrain and the road conditions, which could limit women's actual access or inaccurately classify the facilities that they could more easily access. Research, however, has found that straight-line linkage actually yielded similar results to other geospatial linking methods. ${ }^{45}$

The linkage methodology used in this paper adds to the limited amount of literature on connecting health facility data with population data to understand the relationship between service provision and use. In Haiti, many married women have little or no access to a range of contraceptive methods, which may partially explain the high level of unmet need for family planning and the unbalanced method mix among contraceptive users. The results highlight the importance of offering access to a range of contraceptive methods. The study adds to the findings of other studies showing that making more family planning methods available in health facilities is critical for increasing contraceptive use in both urban and rural areas. In hard-to-reach areas, however, alternative means for supplying contraception, for example through mobile clinics of community-based programmes, should also be considered. Although the focus of this study is married women, the availability of contraceptive method choices would be equally important for the contraceptive use among young unmarried women in Haiti who predominantly rely on male condoms for contraception. 
Acknowledgements The authors acknowledge the financial support from the United States Agency for International Development (USAID) through the DHS Program (\#AID-OAA-C-13-00095).

Contributors WW designed the study, conducted the analysis and drafted the manuscript. LM contributed to the writing and revision of the manuscript. Both authors reviewed and approved the final version.

Funding This study was funded by the United States Agency for International Development (USAID) (Grant Number: \#AID-OAA-C-13-00095).

Competing interests None declared.

Patient consent Not required.

Ethics approval The DHS Program IRB

Provenance and peer review Not commissioned; externally peer reviewed.

Data statement No additional data are available.

Open access This is an open access article distributed in accordance with the Creative Commons Attribution Non Commercial (CC BY-NC 4.0) license, which permits others to distribute, remix, adapt, build upon this work non-commercially, and license their derivative works on different terms, provided the original work is properly cited, appropriate credit is given, any changes made indicated, and the use is non-commercial. See: http://creativecommons.org/licenses/by-nc/4.0

\section{REFERENCES}

1. Ahmed S, Li Q, Liu L, et al. Maternal deaths averted by contraceptive use: an analysis of 172 countries. Lancet 2012;380:111-25.

2. Campbell OMR, Graham WJ, LMSSs group. Strategies for reducing maternal mortality: getting on with what works. The Lancet 2006;368:1284-99.

3. Cleland J, Conde-Agudelo A, Peterson H, et al. Contraception and health. The Lancet 2012;380:149-56.

4. Tsui AO, McDonald-Mosley R, Burke AE. Family planning and the burden of unintended pregnancies. Epidemiol Rev 2010;32:152-74.

5. Darroch JE, Sedgh G, Ball H. Contraceptive technologies. In: Responding to women's needs. New York: Guttmacher Institute, 2011

6. Sedgh G, Ashort LS, Hussain R. Women with an unmet need for contraception in developing countries and their reasons for not using a method. New York: Guttmacher Institute, 2016.

7. Bruce J. Fundamental elements of the quality of care: a simple framework. Stud Fam Plann 1990;21:61-91.

8. Bertrand JT, Sullivan TM, Knowles EA, et al. Contraceptive method skew and shifts in method mix in low- and middle-income countries. Int Perspect Sex Reprod Health 2014;40:144-53.

9. Ho LS, Wheeler E. Using program data to improve access to family planning and enhance the method mix in conflict-affected areas of the democratic republic of the congo. Glob Health Sci Pract 2018;6:161-77.

10. Ross J, Stover J. Use of modern contraception increases when more methods become available: analysis of evidence from 1982-2009. Glob Health Sci Pract 2013:1:203-12.

11. Ross J, Smith E. The family planning effort index: 19992004 and 2009. Washington, DC: Futures Group, Health Policy Initiative, 2010

12. Ross J, Hardee K, Mumford E, et al. Contraceptive method choice in developing countries. Int Fam Plan Perspect 2002;28:32-40.

13. Staveteig S, Mallick L, Winter R. Uptake and discontinuation of longacting reversible contraceptives (LARCs) in low-income countries. Rockville, Maryland, USA: ICF International, 2015.

14. Gage AD, Leslie HH, Bitton A, et al. Assessing the quality of primary care in Haiti. Bull World Health Organ 2017;95:182-90.

15. Skiles MP, Cunningham M, Inglis A, et al. The effect of service environment on demand and use of injectable contraceptives in Malawi. Int Perspect Sex Reprod Health 2015;41:20-30.

16. Tumlinson K, Pence BW, Curtis SL, et al. Quality of care and contraceptive use in Urban Kenya. Int Perspect Sex Reprod Health 2015;41:69.

17. Wang $\mathrm{W}$, Wang $\mathrm{S}$, Pullum $\mathrm{T}$. How family planning supply and the service environment affect contraceptive use: findings from four East African countries. In: DHS analytical studies 26. Calverton, MD: ICF International, 2012

18. Bertrand JT, Ward VM, Santiso-Gálvez R. Family planning in Latin America and the Caribbean: The achievements of 50 years, 2015.
19. Cayemittes M, Busangu MF, Bizimana J. Enquête Mortalité, Morbidité et Utilisation des Services EMMUS-V. 2013. Haiti. Calverton, Maryland: MSPP, IHE et ICF International, 2013.

20. Howse K. Contraceptive Methods by Younger Women: Latin America and Caribbean. Population Horizons Factsheet 2014;9.

21. The World Bank, 2018. Haiti 2015. http://data.worldbank.org/ country/haiti

22. Behrman JA, Weitzman A. Effects of the 2010 Haiti earthquake on women's reproductive health. Stud Fam Plann 2016;47:3-17.

23. Dowell SF, Tappero JW, Frieden TR. Public health in Haiti-challenges and progress. N Engl J Med 2011;364:300-1.

24. Ivers LC. Strengthening the health system while investing in Haiti. Am J Public Health 2011;101:970-1.

25. Gage AJ, Guirlène Calixte M. Effects of the physical accessibility of maternal health services on their use in rural Haiti. Popul Stud 2006;60:271-88.

26. Wang W, Winner M, Burgert-Brucker CR. Limited service availability, readiness, and use of facility-based delivery care in Haiti: a study linking health facility data and population data. Glob Health Sci Pract 2017:5:244-60

27. Fagan T, Dutta A, Rosen J, et al. Family planning in the context of Latin America's universal health coverage agenda. Glob Health Sci Pract 2017;5:382-98.

28. UNFPA. The global program to improve reproductive health commodity security: annual report. New York: UNFPA, 2013.

29. Burgert CR, Colston J, Roy T. Geographic displacement procedure and georeferenced data release policy for the Demographic and Health Surveys. Calverton, Maryland, USA: ICF International, 2013.

30. Burgert CR. Spatial interpolation with demographic and health survey data: key considerations. Rockville, Maryland, USA: ICF International, 2014.

31. Raudenbush SW, Bryk AS. Hiechrarchical linear models: applications and data analysis methods. London: Sage Publications, 2002.

32. Agyei WK, Migadde M. Demographic and sociocultural factors influencing contraceptive use in Uganda. J Biosoc Sci 1995;27:47-60

33. Bakibinga P, Matanda DJ, Ayiko R, et al. Pregnancy history and current use of contraception among women of reproductive age in Burundi, Kenya, Rwanda, Tanzania and Uganda: analysis of demographic and health survey data. BMJ Open 2016;6:e009991.

34. Lasee A, McCormick JB. Demographic and socio-economic determinants of contraceptive use in a low income community of Karachi. J Pak Med Assoc 1996;46:228-31.

35. Stephenson R, Baschieri A, Clements S, et al. Contextual influences on modern contraceptive use in sub-Saharan Africa. Am J Public Health 2007:97:1233-40.

36. Tsui AO, Brown W, Li Q. Contraceptive Practice in Sub-Saharan Africa. Popul Dev Rev 2017;43(Suppl 1):166-91.

37. Wang W, Staveteig S, Winter R. contraceptive use, and unmet need in Sub-Saharan Africa, Latin America, and the Caribbean. Rockville, Maryland, USA: ICF, 2017.

38. Ketende C, Gupta N, Bessinger R. Facility-level reproductive health interventions and contraceptive use in Uganda. Int Fam Plan Perspect 2003;29:130-7.

39. Hanson K, Kumaranayake L, Thomas I. Ends versus means: the role of markets in expanding access to contraceptives. Health Policy Plan 2001;16:125-36.

40. Robinson AL, Seiber EE. Does community clustering mitigate the negative effect of poverty on adolescent condom use in South Africa? Int Fam Plan Perspect 2008;34:121-6.

41. Ministère de la Santé Publique et de la Population (MSPP). Enquête sur les produits et les services de santé reproductive dans 132 institutions sanitaires des 10 départements d'Haït. Tabarre, Haiti: MSPP, 2015.

42. Arends-Kuenning M, Kessy FL. The impact of demand factors, quality of care and access to facilities on contraceptive use in Tanzania. J Biosoc Sci 2007;39:1-26.

43. Blanc AK, Curtis SL, Croft TN. Monitoring contraceptive continuation: links to fertility outcomes and quality of care. Stud Fam Plann 2002;33:127-40.

44. Assaf S, Wang W, Mallick L. Provider counseling and knowledge transfer in health facilities of Haiti, Malawi, and Senegal. Rockville, Maryland, USA: ICF International, 2016

45. Nesbitt RC, Gabrysch S, Laub A, et al. Methods to measure potentia spatial access to delivery care in low- and middle-income countries: a case study in rural Ghana. Int J Health Geogr 2014;13:25. 